\title{
The age and colors of massive white dwarf stars ${ }^{\star}$
}

\author{
L. G. Althaus ${ }^{1,5, \star \star}$, E. García-Berro ${ }^{2,3}$, J. Isern ${ }^{3,4}$, A. H. Córsico ${ }^{1,5, \star \star}$, and R. D. Rohrmann ${ }^{6, \star \star}$ \\ 1 Facultad de Ciencias Astronómicas y Geofísicas, Universidad Nacional de La Plata, Paseo del Bosque s/n, (1900) La Plata, \\ Argentina \\ e-mail: [althaus; acorsico]@fcaglp.unlp.edu.ar \\ 2 Departament de Física Aplicada, Universitat Politècnica de Catalunya, Av. del Canal Olímpic, s/n, 08860 Castelldefels, Spain \\ e-mail: garcia@fa.upc.edu \\ 3 Institut d'Estudis Espacials de Catalunya, Ed. Nexus, c/Gran Capità 2, 08034 Barcelona, Spain \\ e-mail: isern@ieec.fcr.es \\ 4 Institut de Ciències de l'Espai, CSIC, Campus UAB, Facultat de Ciències, Torre C-5, 08193 Bellaterra, Spain \\ 5 Instituto de Astrofísica La Plata, IALP, CONICET \\ ${ }^{6}$ Observatorio Astronómico, Universidad Nacional de Córdoba, Laprida 854, (5000) Córdoba, Argentina \\ e-mail: rohr@oac.uncor.edu
}

Received 17 July 2006 / Accepted 2 January 2007

ABSTRACT

\begin{abstract}
Aims. We present evolutionary calculations and colors for massive white dwarfs with oxygen-neon cores for masses between 1.06 and $1.28 M_{\odot}$. The evolutionary stages computed cover the luminosity range from $\log \left(L / L_{\odot}\right) \approx 0.5$ down to -5.2 .

Methods. Our cooling sequences are based on evolutionary calculations that take into account the chemical composition expected from massive white dwarf progenitors that burned carbon in partially degenerate conditions. The use of detailed non-gray model atmospheres provides us with accurate outer boundary conditions for our evolving models at low effective temperatures.

Results. We examine the cooling age, colors and magnitudes of our sequences. We find that massive white dwarfs are characterized by very short ages to such an extent that they reach the turn-off in their colors and become blue at ages well below 10 Gyr. Extensive tabulations for massive white dwarfs, accessible from our web site, are also presented.
\end{abstract}

Key words. stars: evolution - stars: white dwarfs - stars: interiors

\section{Introduction}

White dwarf stars constitute the most common end-product of stellar evolution. The present population of white dwarfs thus contains precise information about the star formation rate of our Galaxy, as well as about its age, which is information that can be accessible from their mass and luminosity distributions, as long as evolutionary models for the progenitor of white dwarfs and for the white dwarfs themselves are available. However, most of the information about the birth and the evolution of the galactic disk is concentrated at the faint end of the white dwarf luminosity function, which is dominated by the contribution of massive white dwarfs (Díaz-Pinto et al. 1994). It is also worth mentioning at this point that the MACHO collaboration in their first season reported a microlensing event with a duration of 110 days towards the galactic bulge (Alcock et al. 1995). For this particular event a parallax could be obtained from the shape of the light curve, from which a mass of $1.3_{-0.6}^{+1.3} M_{\odot}$ was then derived, indicating that the gravitational lens could possibly be a massive oxygen-neon $(\mathrm{ONe})$ white dwarf or a neutron star.

The interest in very low-luminosity white dwarfs has also increased since the MACHO team proposed that the microlensing events towards the LMC could be due to a population of faint

* Table 1 is only available in electronic form at the CDS via anonymous ftp to cdsarc.u-strasbg.fr $(130.79 .128 .5)$ or via http://cdsweb.u-strasbg.fr/cgi-bin/qcat?/A+A/465/249

$\star \star$ Member of the Carrera del Investigador Científico y Tecnológico, CONICET, Argentina. white dwarfs - see, however, Isern et al. (1998a), Torres et al. (2002), and García-Berro et al. (2004). Since ONe white dwarfs cool faster than the bulk of carbon-oxygen (CO) white dwarfs, it is reasonable to expect that perhaps some of the events could be due to these elusive massive white dwarfs. Moreover, studies about the distribution of masses of the white dwarf population (Finley et al. 1997; Liebert et al. 2005) show the existence of a narrow sharp peak near $0.6 M_{\odot}$, with a tail extending towards higher masses, with several white dwarfs with spectroscopically determined masses within the interval comprised between 1.0 and $1.2 M_{\odot}$.

On the other hand, theoretical evidence suggests that highmass white dwarfs should have cores composed mainly of oxygen and neon - at least for non-rotating stars (Domínguez et al. 1996) - in contrast to average-mass white dwarfs, for which carbon-oxygen cores are expected. The existence of such massive white dwarfs has been suggested as the result of either binary evolution (Marsh et al. 1997) - see also Guerrero et al. (2004) - or of the evolution of heavy-weight intermediate-mass single stars (Ritossa et al. 1996; García-Berro et al. 1997b; Iben et al. 1997; Ritossa et al. 1999). In particular, García-Berro et al. (1997b) found that, when the core mass of a $9 M_{\odot}$ white dwarf progenitor exceeds $\approx 1.05 M_{\odot}$, carbon is ignited off-center in semidegenerate conditions before reaching the thermally pulsing phase at the AGB tip. As a result of repeated carbon-burning shell flashes that ultimately gave rise to carbon exhaustion, these authors found that at the end of carbon burning the star was left with an oxygen-neon core almost devoid of carbon. After 
considerable mass-loss episodes, the progenitor remnant is expected to evolve into the central star of a planetary nebula and ultimately into a white dwarf with an oxygen-neon core. A possible observational counterpart of these ultramassive white dwarfs would be the single massive white dwarf LHS 4033, which has a mass of $\sim 1.32 M_{\odot}$ (Dahn et al. 2004). Other possible massive white dwarfs with oxygen-neon cores would be the magnetic white dwarf PG 1658+441 (Schmidt et al. 1992; Dupuis et al. 2003) - with a mass of $\simeq 1.31 M_{\odot}$ - the highly magnetic white dwarf RE J0317-853 (Ferrario et al. 1997), which has a mass of $\sim 1.35 M_{\odot}$, and the ultramassive white dwarf GD 50 (Dobbie et al. 2006).

The mass-radius relation and the pulsational properties of massive white dwarfs have been the subject of recent theoretical work: Althaus et al. (2005) and Córsico et al. (2004), respectively. However, the evolution (namely, the cooling ages and colors) of massive white dwarfs considering the core composition as predicted by the evolution of massive progenitor stars has not yet been studied in detail, in sharp contrast with the situation for standard CO white dwarfs for which very accurate cooling sequences do exist; see, for instance, Salaris et al. (2000) and references therein. A first attempt to describe the cooling of $\mathrm{ONe}$ white dwarfs was performed by García-Berro et al. (1997a) using a simplified cooling code, but, although the equation of state employed in this work was very detailed, the evolutionary calculations were rather simplistic and the adopted chemical profiles were a flat $\mathrm{ONe}$ mixture, without taking into account the $\mathrm{CO}$ buffer on top of the ONe core that full evolutionary calculations predict. This paper is aimed at precisely filling this gap by presenting new evolutionary calculations for massive white dwarfs with oxygen-neon cores down to very low surface luminosities and effective temperatures. In addition, we present colors and magnitudes for these stars on the basis of non-gray model atmospheres. Detailed model atmospheres also provide us with accurate outer boundary conditions for our evolving models. Our paper is organized as follows. In Sect. 2 we present our input physics. In Sect. 3 we discuss our evolutionary sequences. Finally, in the last section we summarize our findings and draw our conclusions.

\section{Input physics}

The evolutionary code and the starting white dwarf configurations used in this work are essentially those used recently in the calculation of mass-radius relations for massive white dwarfs by Althaus et al. (2005), and we refer the reader to that paper for further details. Because we are now interested in providing accurate cooling times, some major improvements to the input physics assumed in Althaus et al. (2005) have been made. First, we have included the release of latent heat upon crystallization. Despite the fact that crystallization in massive white dwarfs takes place at higher stellar luminosities than do standard carbon-oxygen white dwarfs, its impact on the cooling times is not entirely negligible. In our calculations, crystallization sets in when the ion coupling constant $\Gamma=180$, where $\Gamma \equiv Z^{2} \mathrm{e}^{2} / \bar{r} k_{\mathrm{B}} T$ and $\bar{r}$ is the radius of the Wigner-Seitz sphere. We considered a latent heat release of $k_{\mathrm{B}} T$ per ion, which was spread over the range $175<\Gamma<185$. Phase separation upon crystallization has been shown to introduce negligible time delays for massive white dwarfs (García-Berro et al. 1997a) and, consequently, was not taken into account. Second, for the high-density regime, we adopted the equation of state described in Segretain et al. (1994) to account for all the important contributions for both the liquid and the solid phases. In particular, this equation of state, besides the contribution from partially degenerate electrons, accounts for the exchange contribution (Stringfellow et al. 1990) and the quantum (diffraction) corrections (Hansen \& Vieillefosse 1975) in the fluid phase, as well as the harmonic contribution (Chabrier 1993), the anaharmonic contribution (Stringfellow et al. 1990), and the electrostatic contribution (Stringfellow et al. 1990) in the solid phase. For the electrons the exchange effects at finite temperature (Kovetz et al. 1972), the correlation contribution (Nozières \& Pines 1958), and the electron-ion coupling contribution (Yakovlev \& Shalibkov 1989) were also taken into account. For the low-density regime, we used an updated version of the equation of state of Magni \& Mazzitelli (1979). Neutrino emission rates for pair, photo, plasma, and bremsstrahlung processes and for high-density conductive opacities were taken from Itoh et al. (1994, 1996a, 1996b). Convection was treated in the framework of the mixing length theory as given by the ML2 parameterization (Tassoul et al. 1990). Radiative opacities were those of OPAL (Iglesias \& Rogers 1996), complemented at low temperatures with the Alexander \& Ferguson (1994) molecular opacities.

The initial white dwarf models from which we started our calculations of the cooling sequences correspond to hot white dwarf configurations with a chemical stratification appropriate to massive white dwarfs resulting from progenitor stars with solar metallicity that are expected to have burned carbon in semidegenerate conditions - see, for instance Ritossa et al. (1996). According to the calculations of these authors, the core is mainly composed of ${ }^{16} \mathrm{O}$ and ${ }^{20} \mathrm{Ne}$, plus some traces of ${ }^{12} \mathrm{C},{ }^{23} \mathrm{Na}$, and ${ }^{24} \mathrm{Mg}$ and is the result of repeated shell flashes that take place during the carbon-burning phase in massive intermediate-mass stars (García-Berro et al. 1997b; Gil-Pons et al. 2003). The core chemical profiles of our models are flat throughout the core. Such profiles are expected if Rayleigh-Taylor instabilities act to smooth-out regions with negative molecular weight gradients. The outer layer chemical stratification consists of a pure hydrogen envelope of $10^{-6} M_{*}$ (plus a small inner tail) overlying a helium-dominated shell of $4 \times 10^{-4} M_{*}$ and, below that, a buffer rich in ${ }^{12} \mathrm{C}$ and ${ }^{16} \mathrm{O}$. The amount of hydrogen we adopted is an upper limit as imposed by nuclear reactions. All the evolutionary sequences considered in this work have the same core composition and shell profile, which remains fixed during the evolutionary sequences and corresponds to that illustrated in Fig. 4 of Córsico et al. (2004). The shape of the outer layer chemical profile is given by element diffusion at low luminosities. Nevertheless, diffusion was switched off in the present calculations. Although minor changes in the chemical profile are expected because of the different masses of progenitor objects, we believe that these had a negligible influence on the cooling times of our sequences.

We computed the evolution of our models in a self-consistent way, using detailed non-gray model atmospheres, which also allow us to derive color indices and magnitudes of these white dwarfs. Our model atmospheres are based on an ideal equation of state. The following species have been considered: $\mathrm{H}, \mathrm{H}_{2}, \mathrm{e}^{-}$, $\mathrm{H}^{-}, \mathrm{H}^{+}, \mathrm{H}_{2}{ }^{+}$, and $\mathrm{H}_{3}{ }^{+}$. All the relevant bound-free, free-free, and scattering processes contributing to opacity were included in our calculations. At low $T_{\text {eff }}$ values, collision-induced absorption (CIA) from molecular hydrogen due to collisions with $\mathrm{H}_{2}$ represents a major source of opacity in the infrared and dominates the shape of the emergent spectrum. Collision-inducedabsorption cross sections are from Borysow et al. (1997). Broadband color indices have been calculated using the optical $B V R I$ and infrared $J H K$ passbands of Bessell (1990) and Bessell $\&$ Brett (1988), respectively, with calibration constants from 
Bergeron et al. (1997). More specific details about the input physics and computational issues of the model atmospheres used in this work are described at length in Rohrmann (2001). However, for the purpose of this paper it is important to realize that in order to compute the outer boundary conditions for our evolving models, the values of the pressure and the temperature at the bottom of the atmosphere are required. In the calculations reported here non-gray model atmopheres are fully integrated each time the outer boundary conditions must be evaluated as evolution proceeds.

The initial stellar models were derived from an artificial evolutionary procedure starting from the full evolution of a $0.95 M_{\odot}$ white dwarf model; see Althaus et al. (2005) and references cited therein. Despite the correctness of our artificial procedure to generate starting white dwarf configurations, model ages corresponding to the very first computed stages of evolution should be taken with some care. However, for ages over $10^{5} \mathrm{yr}$, the evolutionary calculations presented here are independent of the initial conditions. We computed the evolution of massive white dwarf models with stellar masses of 1.06, 1.10, 1.16, 1.20, 1.24, and $1.28 M_{\odot}$. The lower limit of this mass range corresponds to the approximate minimum mass for a white dwarf to have an ONe core. The precise value of this mass is still not well known. White dwarfs with masses higher than $\sim 1.1 M_{\odot}$ have progenitors that proceed through carbon burning (García-Berro et al. 1997b), whereas white dwarfs with masses lower than $\sim 1.0 M_{\odot}$ have progenitors that never ignite carbon (Salaris et al. 1997). The evolutionary stages computed cover the luminosity range from $\log \left(L / L_{\odot}\right) \approx 0.5$ down to -5.2 .

In addition to the calculation of the evolution of white dwarf sequences with pure hydrogen envelopes, we computed the evolution of the same sequences mentioned above but for the case of pure helium envelopes. In this case, we assumed a grey atmosphere. Although the employment of a gray atmosphere in our helium envelope sequences prevents a precise quantitative comparison with the case of hydrogen envelope sequences, this still gives us a reasonable assessment of the cooling times for these helium envelope sequences.

\section{Evolutionary results}

We begin by examining Fig. 1, which shows the evolutionary times as a function of the surface luminosity for the 1.06, 1.10, $1.16,1.20,1.24$, and $1.28 M_{\odot}$ ONe white dwarf models with pure $\mathrm{H}$ atmospheres. The figure also shows (i) the locii along the curves where the neutrino emission luminosity becomes 100 and $10 \%$ of the surface luminosity, (ii) the onset of core crystallization (at $\Gamma=180$ ), and (iii) the dimensionless Debye temperature at the core when it reaches $\theta_{\mathrm{D}} / T=20$, where the Debye temperature is $\theta_{\mathrm{D}}=3.48 \times 10^{3}\langle Z / A\rangle \rho^{1 / 2}$. Several physical processes that take place along the evolution leave their signatures in the cooling curve. During the first stages of evolution, the interior temperature is relatively high (between $T \sim 1.2$ and $1.5 \times 10^{8} \mathrm{~K}$, depending on the mass of the white dwarf) and neutrino emission constitutes a powerful sink of energy that considerably affects both the cooling timescales of massive white dwarfs and, as shown in Althaus et al. (2005), also the mass-radius relation of these stars. Indeed, neutrino emission luminosity far exceeds the photon luminosity during the hot white dwarf stages. As neutrino emission gradually decreases, this accelerated cooling phase arrives at its end and the slope of the cooling curve changes, as can be seen in Fig. 1. Note that neutrino losses persist further down to luminosities $\log \left(L / L_{\odot}\right) \sim-1$, but their effects on the cooling curve are negligible at those evolutionary stages.

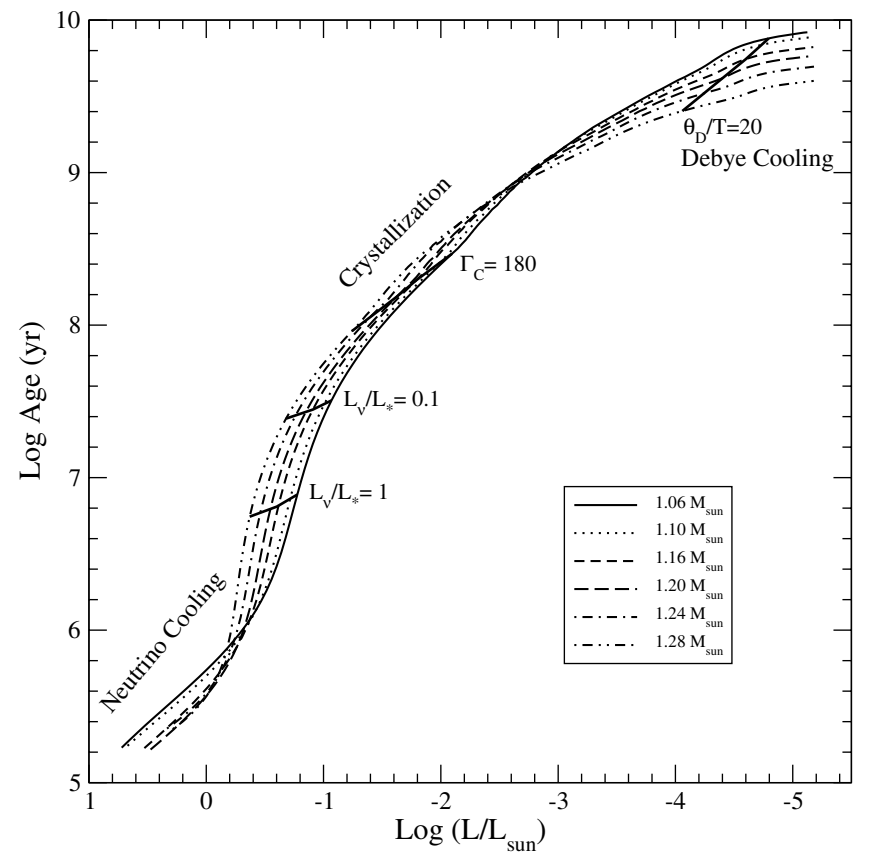

Fig. 1. Evolutionary times as a function of the surface luminosity for our massive white dwarf models with pure $\mathrm{H}$ atmospheres. The stages for which neutrino emission, crystallization and Debye cooling drive the evolution are indicated. The locii along the curves where the neutrino contribution to the surface luminosity (100 and 10\%), the onset of core crystallization (at $\Gamma=180$ ) and when the dimensionless Debye temperature at the core reaches $\theta_{\mathrm{D}} / T=20$ are clearly indicated. Note the relatively short ages characterizing these models at advanced stages of evolution, particularly for the most massive ones.

The release of latent heat upon crystallization is known to influence the evolutionary times of white dwarfs as well. However, in the case of massive ONe white dwarfs, the crystallization of the core is a process that occurs at relatively high luminosities $\log \left(L / L_{\odot}\right)=-2.15$ and -1.30 for the 1.06 and $1.28 M_{\odot}$ cooling sequences, respectively (see Fig. 1) - and its impact on the evolution of the star therefore remains moderate. It is thus important to note that crystallization profoundly influences the cooling behavior of only the less massive sequences.

The short ages that characterize our white dwarf models at very low surface luminosities is a noteworthy feature. This is particularly noticeable for our most massive models. For instance, the $1.28 M_{\odot}$ cooling sequence reaches a luminosity of $\log \left(L / L_{\odot}\right)=-5.15$ in only 4 Gyr. In fact our results indicate that the most massive white dwarfs could be, depending on the distance, unobservable at ages well below $10 \mathrm{Gyr}$ with the current observational facilities. For instance, if there are such massive cool white dwarfs at the distance of say the Hyades, the apparent magnitude would only be $m_{V} \sim 21.5$ or so, and at $1 \mathrm{kpc}$, $m_{V} \sim 28$, whereas the $K$-band apparent magnitudes would be 1 or 2 magnitudes brighter, according to our models. At these distances such massive cool white dwarfs might be observable with present or near future facilities but the observations would be very challenging. At the lowest computed luminosities, our massive white dwarf models experience the development of the socalled fast Debye cooling. In this sense, we would like to mention at this point of the discussion that, for the $1.28 M_{\odot}$ model at $\log \left(L / L_{\odot}\right)=-4.95$, the dimensionless Debye temperature remains well above 10 for about the $95 \%$ of the structure (that is, most of the star is within the Debye regime), with the consequence that the thermal content goes rapidly to zero in this 


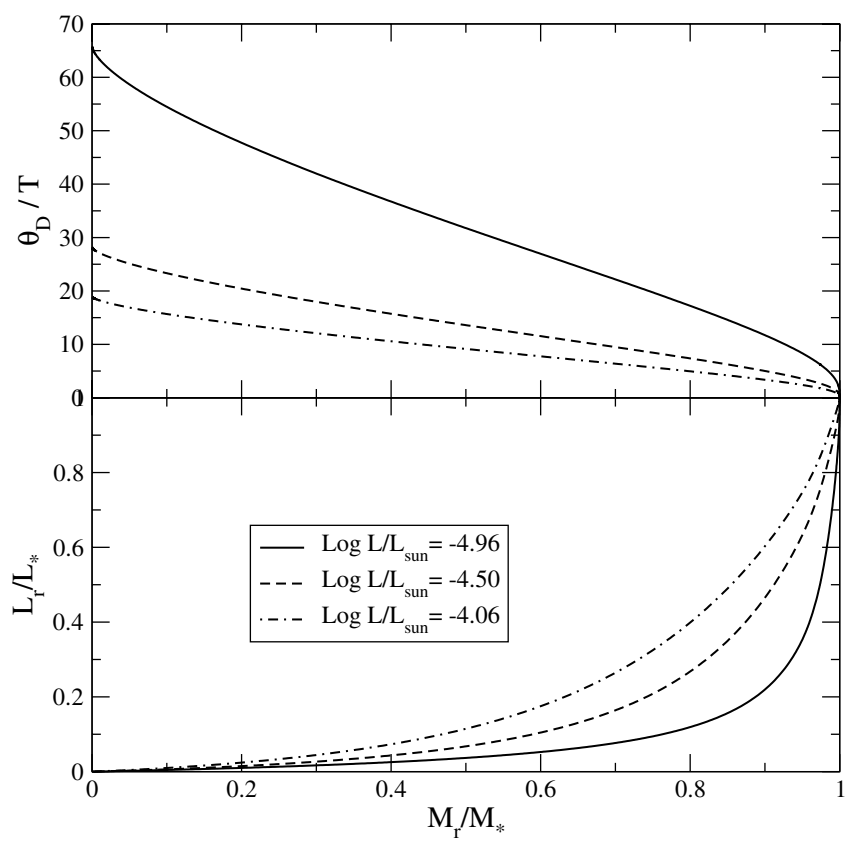

Fig. 2. Upper panel: the dimensionless Debye temperature in terms of the mass fraction for three selected low-luminosity $1.28 M_{\odot}$ models. Bottom panel: internal luminosity versus the mass fraction for the same models shown in the upper panel. Note that most of the structure of the lowest luminosity model is well inside the Debye regime.

region. For less massive $\mathrm{CO}$ white dwarfs, this takes place at luminosities of about $\log \left(L / L_{\odot}\right)=-6$ (D'Antona \& Mazzitelli 1989). This fact is reflected in Fig. 2, which displays the run of the Debye temperature and the fractional luminosity for some selected low-luminosity stages of the $1.28 M_{\odot}$ model sequence. Note that for the coolest models, the luminosity output is almost negligible in the inner regions of the star and that only in the outermost layers are the ratio $\theta_{\mathrm{D}} / T$ and the degeneracy parameter not very large. Hence, these layers can still be compressionally heated, making the main energy source of the star. In fact, the compression of the outermost layers provides the bulk of the star luminosity; see Isern et al. (1998b) for a detailed description of the cooling of white dwarfs.

For a better understanding of the physical processes occurring in the interior of our massive white dwarf models we show in Fig. 3 the run of the central temperatures versus ages and surface luminosities (left and right panel, respectively) for the 1.06, 1.16 and $1.28 M_{\odot}$ model sequences. The changes in slope of the $\log T_{\mathrm{c}}$ versus $\log \left(L / L_{\odot}\right)$ diagram at both high luminosities and at the low luminosity end are noticeable. At high luminosity, it reflects the end of neutrino dominated regime. At low luminosity, the increase in the slope of the curve occurs when the hydrogenrich outer convection zone approaches the isothermal degenerate core (see Fig. 4). As a result, the white dwarf has additional thermal energy to radiate (D’Antona \& Mazzitelli 1989). This helps to understand the lengthening of the evolutionary cooling times occurring at $\log \left(L / L_{\odot}\right) \approx-4$ in Fig. 1 .

Non-negligible differences in the cooling of white dwarfs arise from the different thicknesses of the $\mathrm{H}$ envelopes with which white dwarfs proceed during their cooling track. To assess such differences, we considered the evolution of massive white dwarfs for the extreme situation of pure helium envelopes. The resulting evolutionary times are displayed in Fig. 5. At advanced stages in the evolution, the central temperature of the models is strongly tied to the details of the outer layer's chemical
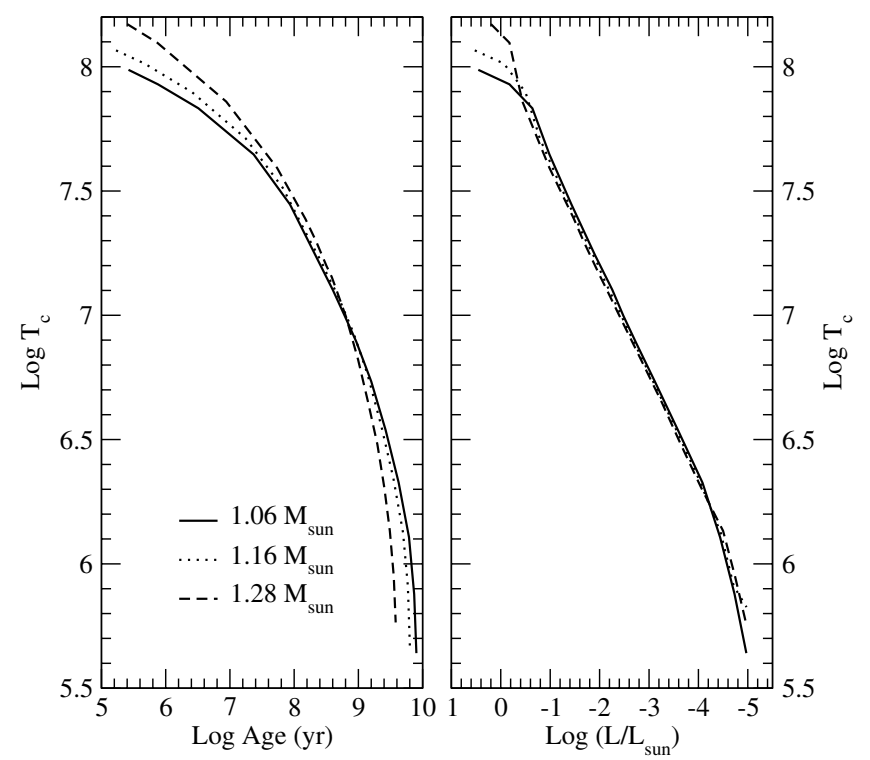

Fig. 3. Central temperature versus age and surface luminosity as a function of the age (left and right panels, respectively) for the 1.06, 1.16, and $1.28 M_{\odot}$ sequences.

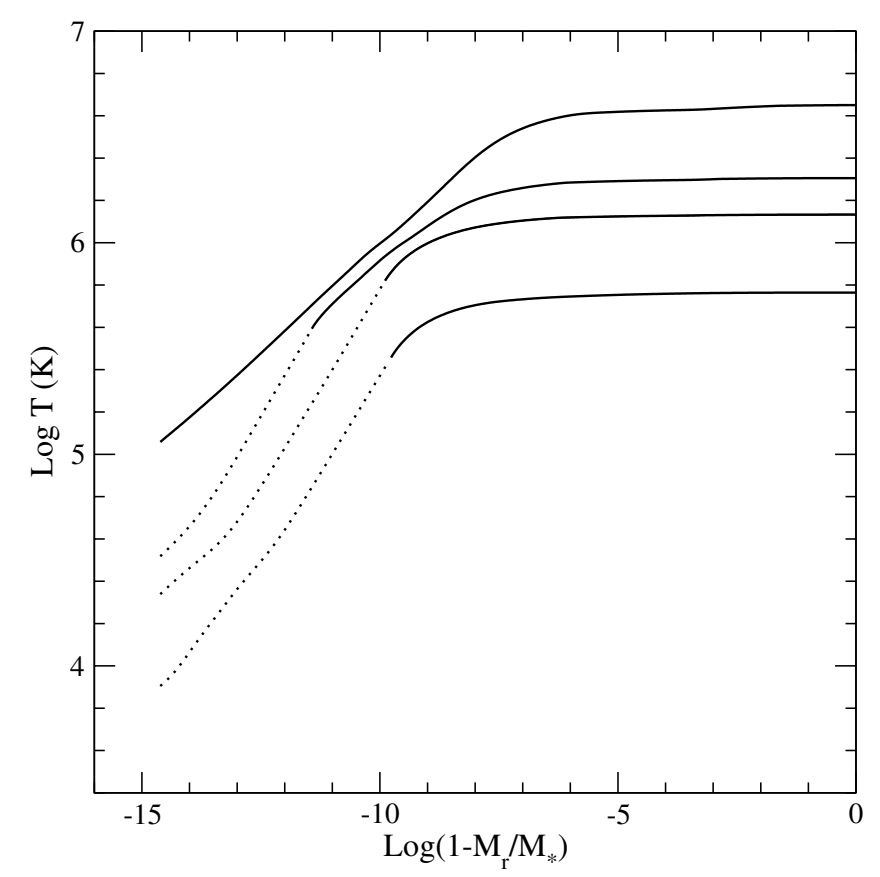

Fig. 4. The run of temperature in terms of the outer mass fraction for the $1.28 M_{\odot}$ model at luminosities $\log \left(L / L_{\odot}\right)=-3.25,-4.05,-4.50$, and -4.96 (from top to bottom). Dotted lines represent convectively unstable layers.

stratification. This fact starts to manifest itself when the boundary of the degenerate core reaches the base of the $\mathrm{H}$ envelope at $\log \left(L / L_{\odot}\right) \sim-2.5$; see Tassoul et al. (1990) for details. As a result, white dwarfs with helium envelopes (and hence more transparent) evolve more rapidly than those white dwarfs with $\mathrm{H}$ envelopes, as is clear by examining Fig. 5. As expected, the helium sequences will reach the Debye cooling conditions earlier than their hydrogen counterparts (compare Figs. 1 and 5). Note that the $1.28 M_{\odot}$ white dwarf cooling sequence with a helium atmosphere needs only 2.24 Gyr to reach $\log \left(L / L_{\odot}\right)=-4.70$, a factor about 1.5 less than the age required by the pure $\mathrm{H}$ counterpart. At 


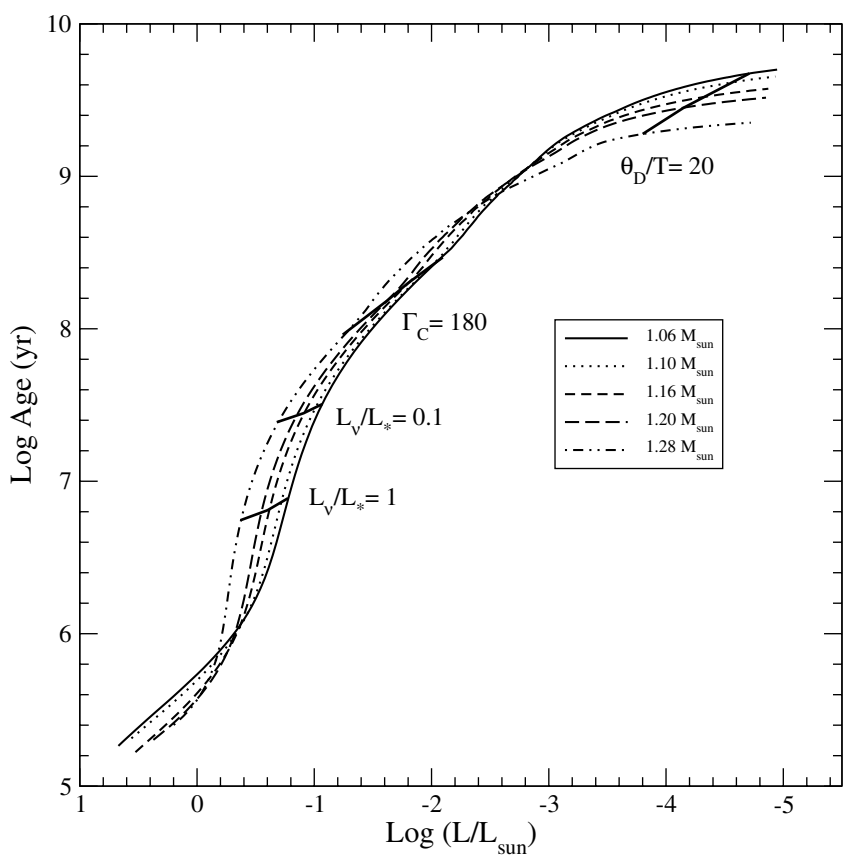

Fig. 5. Same as Fig. 1 but for white dwarf models with pure He atmospheres.

ages below $5 \mathrm{Gyr}$, most of our cooling sequences with pure $\mathrm{He}$ atmospheres will have cooled down to below $\log \left(L / L_{\odot}\right) \approx-5.5$.

The fast evolution of our massive white dwarfs at low surface luminosities raises the possibility that these white dwarfs with pure $\mathrm{H}$ atmospheres reach the turn-off in their colors and become blue afterwards within relatively short ages. To elaborate this point further, we show in Fig. 6 the evolution of our pure $\mathrm{H}$ white dwarf cooling sequences in the $(B-V, V-K)$ color-color diagram. As a result of the collision-induced absorption from molecular hydrogen, a process that reduces the infrared flux and forces radiation to emerge at higher frequencies, very cool white dwarfs are expected to become bluer as they age (Hansen 1998). As shown in Fig. 6, this effect is also present in our massive white dwarf sequences. Indeed, all our sequences exhibit a pronounced turn-off in their color indices at advanced stages of evolution and become bluer with further evolution. In particular, below $4500 \mathrm{~K}$, massive white dwarfs become markedly bluer in the $(B-V, V-K)$ two-color diagram. The remarkable point is that the turn to the blue happens within cooling times much shorter than 10 Gyr. If we extrapolate our results, we should thus expect dim massive white dwarfs characterized by relatively short ages to exhibit blue colors.

The molecular hydrogen absorption at low effective temperatures also affects the evolution of our models in the colormagnitude diagram, as shown in Figs. 7 and 8, which display the run of the absolute visual magnitude $M_{V}$ in terms of the $V-K$ and $V-I$ color indices, respectively. For the evolutionary stages computed in this work, the turn to the blue is noticeable for the $V-K$ color index. Note that in this diagram, all our sequences are expected to become markedly blue for relatively short ages. Specifically, our sequences have cooling ages between 3.7 and 6.8 Gyr (depending on the stellar mass value) at the turn-off point, which occurs at $M_{V} \approx 17$. For later stages, Debye cooling is dominant and evolution indeed proceeds very quickly. Note as well that in the $\left(M_{V}, V-K\right)$ diagram our sequences remain brighter than $M_{V} \approx 18$ at advanced stages. For the lowest luminosities we have driven our cooling tracks, our sequences have

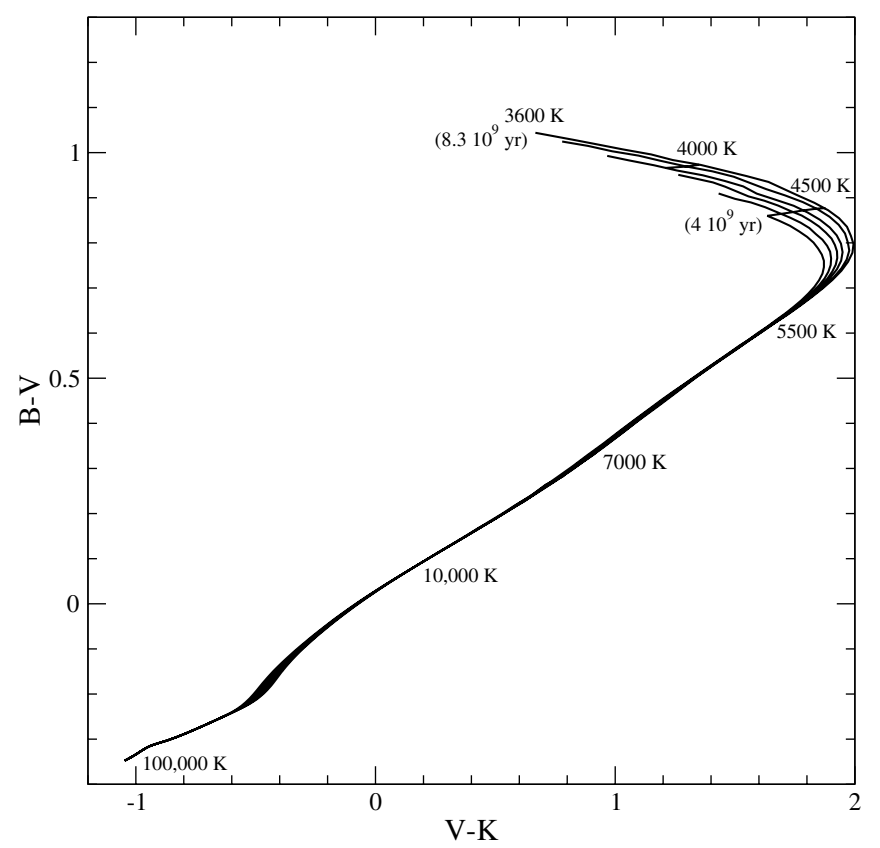

Fig. 6. $(B-V, V-K)$ color-color diagram for our massive white dwarf cooling sequences with masses $1.06,1.10,1.16,1.20,1.24$, and $1.28 M_{\odot}$ (from top to bottom). Lines tracing the locii of equal effective temperatures are labelled with the corresponding values. For the 1.06 and $1.28 M_{\odot}$ sequences, the ages at $T_{\text {eff }}=3600$ and $4500 \mathrm{~K}$, respectively, are also indicated. Note that all the cooling sequences exhibit a pronounced turn-off at advanced stages of evolution. Below $4500 \mathrm{~K}$, massive white dwarfs become markedly blue in this diagram within cooling times much less than $10 \mathrm{Gyr}$.

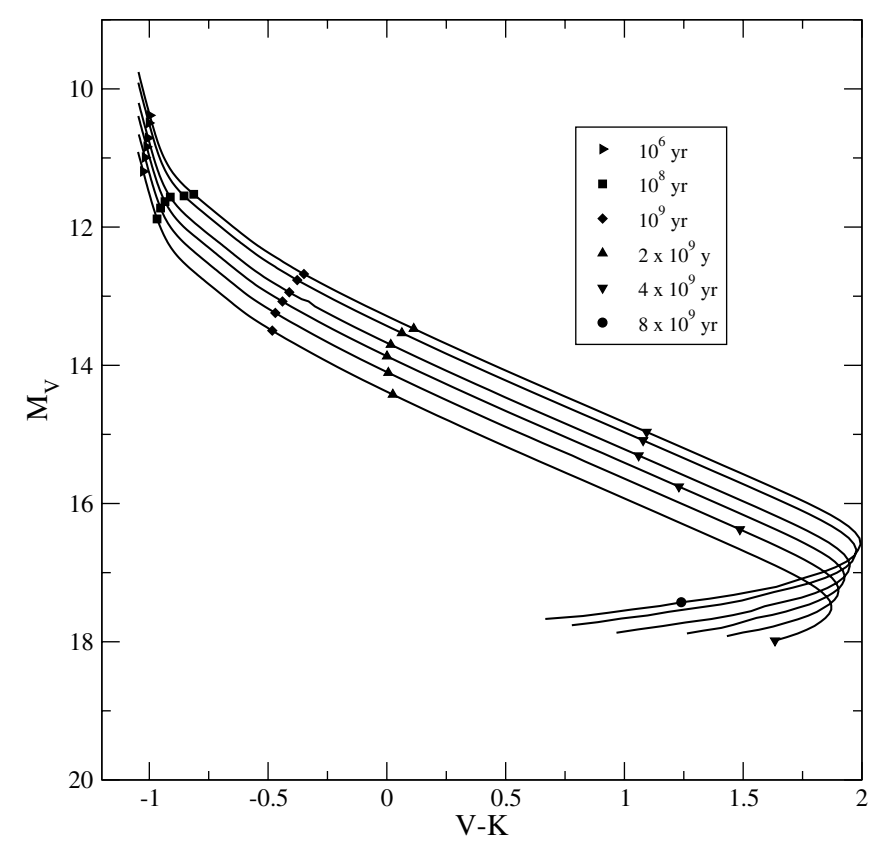

Fig. 7. Absolute visual magnitude $M_{V}$ in terms of the color index $V-K$ for our massive white dwarf sequences with masses 1.06, 1.10, 1.16, $1.20,1.24$ and $1.28 M_{\odot}$ (from top to bottom). On each curve, filled symbols represent cooling ages, as indicated within the inset. The pronounced turn-off at advanced stages of the evolution is apparent. Note that for ages well within $10 \mathrm{Gyr}$, all our sequences are expected to become markedly blue in this diagram.

not yet reached the turn-off point in the $\left(M_{V}, V-I\right)$ diagram, as inferred from Fig. 8. For the turn to the blue to occur, the 


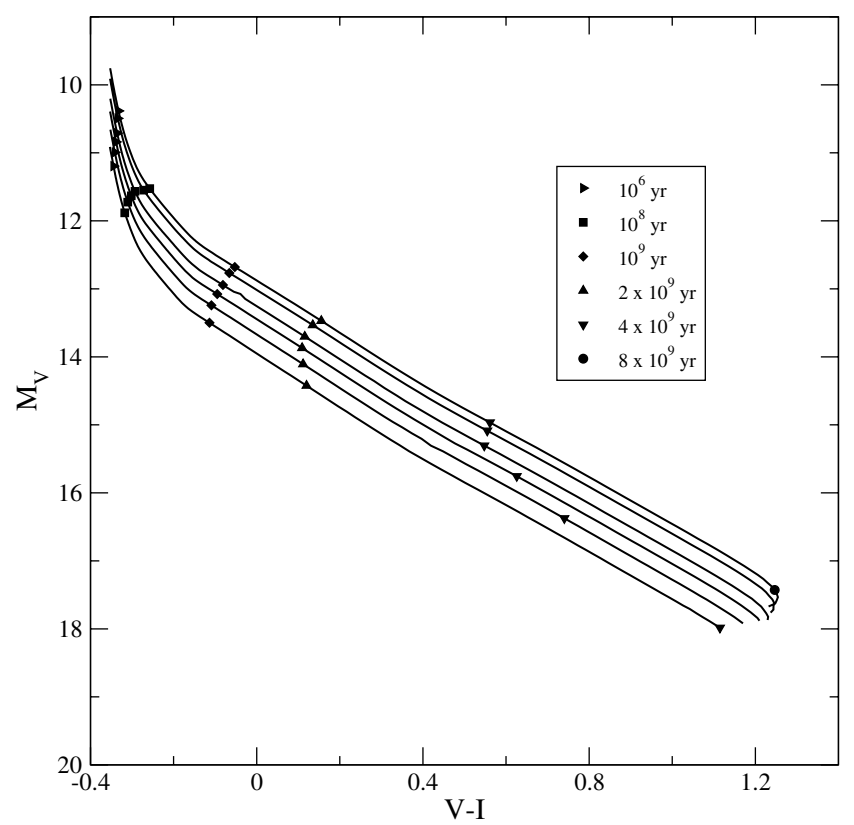

Fig. 8. Same as Fig.7 but for the color index $V-I$. Unlike the $V-K$, this index has not yet reached the turn-off point even for the coolest computed models.



Fig. 9. Absolute visual magnitude as a function of the effective temperature for our massive pure $\mathrm{H}$ white dwarf cooling sequences with masses of (from top to bottom) 1.06, 1.10, 1.16, 1.20, 1.24, and $1.28 M_{\odot}$. The thin lines plot isochrones at the labelled ages. Observational data for some massive H-rich white dwarfs from Bergeron et al. (2001) and Liebert et al. (2005) are included - squares and stars, respectively.

evolution should have proceeded to lower effective temperatures than those computed here. Although this would certainly take place in ages shorter than $10 \mathrm{Gyr}$, the expected surface luminosity of the models would be extremely low to be detected - below $\log \left(L / L_{\odot}\right)=-5.5$.

In Fig. 9 we illustrate the run of the absolute visual magnitude as a function of the effective temperature for our massive pure $\mathrm{H}$ white dwarf sequences. In addition, we draw various isochrones at ages of $0.01,0.1,1,2$, and $4 \mathrm{Gyr}$. It is clear

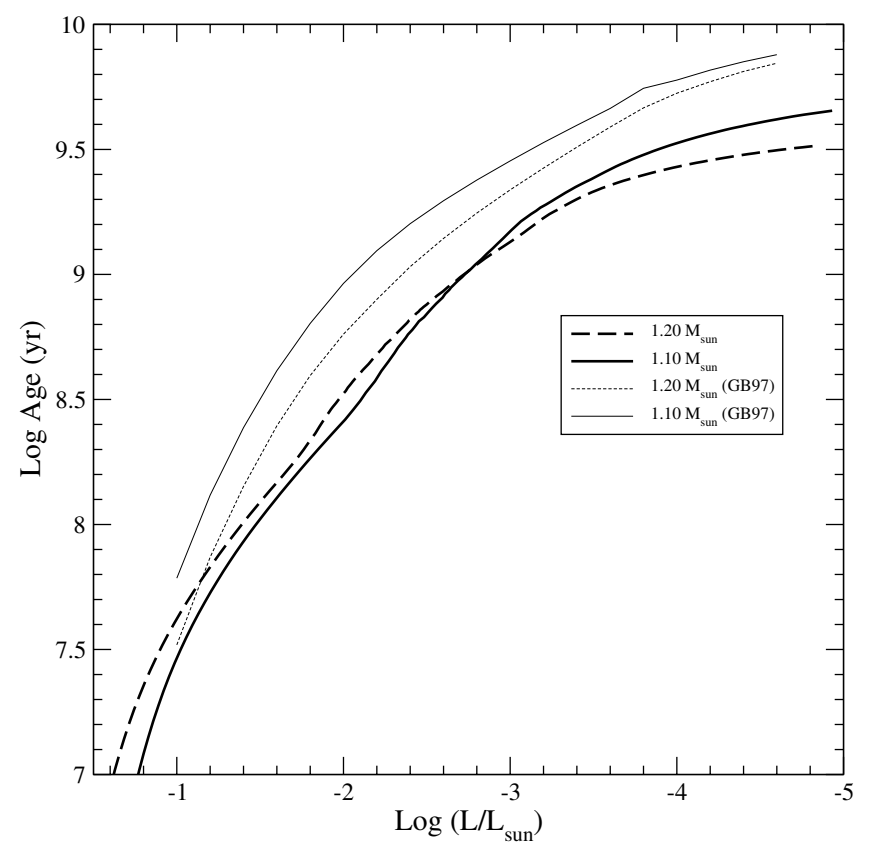

Fig. 10. Comparison between our 1.10 and $1.20 M_{\odot}$ sequences (helium atmospheres) with those of García-Berro et al. (1997a).

that the coolest white dwarf models are not necessarily the oldest. For instance, in $4 \mathrm{Gyr}$ the $1.28 M_{\odot}$ models have cooled down to $4600 \mathrm{~K}$, while the $1.06 M_{\odot}$ model sequence remains much hotter $(7000 \mathrm{~K})$ at this age. Observational data for massive white dwarfs with $\mathrm{H}$-rich atmospheres taken from Bergeron et al. (2001) and Liebert et al. (2005) are also included in the figure. As compared with the ages quoted by the mentioned authors, our results suggest younger ages for the white dwarfs in Fig. 9. For instance, for WD 1658+441, which, according to our calculations would have an appreciable fraction of its core in a crystallized state, we derive an age of $0.28 \mathrm{Gyr}$, as compared with the 0.38 Gyr quoted by Liebert et al. (2005). The shorter ages are due partly to the abundant ${ }^{20} \mathrm{Ne}$ in the core of our massive models, which results in a lower specific heat per gram.

Finally, in Fig. 10 we compare our 1.10 and $1.20 M_{\odot}$ sequences with helium atmospheres with those of García-Berro et al. (1997a) for ONe cores. Note that our calculations predict much younger ages than those derived in García-Berro et al. (1997a). In part, the differences between both sets of calculations have their origin in the different chemical abundance distributions characterizing the pertinent models. For instance, our models are characterized by a helium-dominated shell that is four times more massive than considered in García-Berro et al. (1997a). It should also be taken into account that the preliminary calculations of García-Berro et al. (1997a) were done using a simplified model that obtain the evolution from calculating the binding energy very accurately (actually using the same equation of state employed here) and coupling it with a relationship linking the surface luminosity with the core temperature of an otherwise typical CO white dwarf of mass $0.6 M_{\odot}$.

In closing, we list the main characteristics of our 1.06, 1.16, and $1.28 M_{\odot}$ white dwarf sequences in Table 1 . Specifically, we list the effective temperature, the surface gravity, the age, the colors, and the absolute visual magnitude. 


\section{Conclusions}

We have computed the evolution of massive white dwarf stars with oxygen-neon cores for masses ranging from 1.06 to $1.28 M_{\odot}$, which covers the expected range of masses for which these stars should presumably exist. The evolutionary tracks for both pure $\mathrm{H}$ and pure He envelopes were computed for surface luminosities spanning the range from $\log \left(L / L_{\odot}\right) \approx 0.5$ down to -5.2 . The use of detailed non-gray model atmospheres provides us with accurate outer boundary conditions for our evolving models at low effective temperatures. To our knowledge this is the first attempt to compute the evolution of massive white dwarfs with a realistic equation of state - which includes all the non-ideal, corrective terms, and the full temperature dependence - and reliable chemical profiles for the degenerate interior expected from the previous evolutionary history of massive white dwarf progenitors that burned carbon in semidegenerate conditions. We have examined the cooling ages, colors, and magnitudes of our sequences and find that massive white dwarfs are characterized by very rapid evolution. Indeed, at still observable luminosities, we find that the cooling of massive white dwarfs is largely dominated by Debye cooling with the result that these white dwarfs could be unobservable at ages below the age of the Galactic disk with the current observational facilities. At such advanced stages, we find our sequences to have reached the turnoff in their colors and thus become blue in short times.

The results presented here will be helpful in interpreting recent observations of white dwarfs with very high surface gravities (Dahn et al. 2004; Madej et al. 2004; Nalezyty \& Madej 2004), which up to now rely on previous evolutionary sequences that were computed assuming carbon-oxygen cores. Finally, we prepared detailed tabulations of ages, colors, and magnitudes for all our white dwarf sequences, which are available at our web site: http: //www . fcaglp. unlp. edu . ar/evolgroup/.

Acknowledgements. We acknowledge the valuable report of our referee, G.S. Stringfellow, which strongly improved both the scientific content and presentation of the paper. This research was supported by the MCYT grant AYA0508013-C03-01 and 02, by the European Union FEDER funds, by the AGAUR and by the PIP 6521 grant from CONICET.

\section{References}

Alcock, C., Allsman, R. A., Alves, D., et al. 1995, ApJ, 454, L125

Alexander, D. R., \& Ferguson, J. W. 1994, ApJ, 437, 879

Althaus, L. G., García-Berro, E., Isern, J., \& Córsico, A. H. 2005, A\&A, 441, 689

Bergeron, P., Ruiz, M. T., \& Legget, S. K. 1997, ApJS, 108, 339
Bergeron, P., Legget, S. K., \& Ruiz, M. T. 2001, ApJS, 133, 413

Bessell, M. S. 1990, PASP, 102, 1181

Bessell, M. S., \& Brett J. M. 1988, PASP, 100, 1134

Borysow, A., Jorgensen, U. G., \& Zheng, C. 1997, A\&A, 324, 185

Chabrier, G. 1993, ApJ, 414, 695

Córsico, A. H., García-Berro, E., Althaus, L. G., \& Isern, J. 2004, A\&A, 427, 923

Dahn, C. C., Bergeron, P., Liebert, J., et al. 2004, ApJ, 605, 400

D’Antona, F., \& Mazzitelli, I. 1989, ApJ, 347, 934

Díaz-Pinto, A., García-Berro, E., Hernanz, M., Isern, J., \& Mochkovitch, R. 1994, A\&A, 282, 86

Dobbie, P. D., Napiwotzki, R., Lodieu, N., et al. 2006, MNRAS, in press

Domínguez, I., Straniero, O., Tornambé, A., \& Isern, J. 1996, ApJ, 472, 783

Dupuis, J., Chayer, P., Vennes, S., Allard, N.F ., \& Hébrard, G. 2003, ApJ, 598, 486

Ferrario, L., Vennes, S., Wickramasinghe, D. T., Bailey, J. A., \& Christian, D. J. 1997, MNRAS, 292, 205

Finley, D. S., Koester, D., \& Basri, G. 1997, ApJ, 488, 375

García-Berro, E., Isern, J., \& Hernanz, M. 1997a, MNRAS, 289, 973

García-Berro, E., Ritossa, C., \& Iben, I. 1997b, ApJ, 485, 765

García-Berro, E., Torres, S., Isern, J., \& Burkert, A. 2004, A\&A, 418, 53

Gil-Pons, P., García-Berro, E., José, J., Hernanz, M., \& Truran, J. W. 2003, A\&A, 407, 1021

Guerrero, J., García-Berro, E., \& Isern, J. 2004, A\&A, 413, 257

Hansen, B. M. S. 1998, Nature, 394, 860

Hansen, J. P., \& Vieilleifosse, P. 1975, Phys. Lett. A, 53, 187

Iben, I., Ritossa, C., \& García-Berro, E. 1997, ApJ, 489, 772

Iglesias, C. A., \& Rogers, F. 1996, ApJ, 464, 943

Isern, J., García-Berro, E., Hernanz, M., Mochkovitch, R., \& Torres, S. 1998a, ApJ, 503, 239

Isern, J., García-Berro, E., Hernanz, M., \& Mochkovitch, R. 1998b, J. Phys.: Cond. Matt., 49, 11263

Itoh, N., Hayashi, H., \& Kohyama, Y. 1994, ApJ, 416, 438

Itoh, N., Nishikawa, A., \& Kohyama, Y. 1996a, ApJ, 470, 101

Itoh, N., Hayashi, H., Nishikawa, A., \& Kohyama, Y. 1996b, ApJS, 102, 41

Kovetz, A., Lamb, D. Q., \& Van Horn, H. M. 1972, ApJ, 174, 109

Liebert, J., Bergeron, P., \& Holberg, J. 2005, ApJS, 156, 47

Madej, J., Nalezyty, M., \& Althaus, L. G. 2004, A\&A, 419, L5

Magni, G., \& Mazzitelli, I. 1979, A\&A, 72, 134

Marsh, M. C., Barstow, M. A., Buckley, D. A., et al. 1997, MNRAS, 287, 705

Nalezyty, M., \& Madej, J. 2004, A\&A, 420, 507

Nozières, P., \& Pines, D. 1958, Phys. Rev., 111, 1442

Ritossa, C., García-Berro, E., \& Iben, I. 1996, ApJ, 460, 489

Ritossa, C., García-Berro, E., \& Iben, I., 1999, ApJ, 515, 381

Rohrmann, R. D. 2001, MNRAS, 323, 699

Salaris, M., Domínguez, I., García-Berro, E., et al. 1997, ApJ, 486, 413

Salaris, M., García-Berro, E., Hernanz, M., \& Isern, J. 2000, ApJ, 544, 1036

Schmidt, G. D., Bergeron, P., Liebert, J., \& Saffer, R. A. 1992, ApJ, 394, 603

Segretain, L., Chabrier, G., Hernanz, M., García-Berro, E., \& Isern, J. 1994, ApJ, 434,641

Stringfellow, G. S., De Witt, H. E., \& Slattery, W. I., 1990, Phys. Rev. A, 41, 1105

Tassoul, M., Fontaine, G., \& Winget, D. E., 1990, ApJS, 72, 335

Torres, S., García-Berro, E., Burkert, A., \& Isern, J. 2002, MNRAS, 336, 971

Yakovlev, D. G., \& Shalybkov, D. A. 1989, Astrophys. Space Phys. Rev., 7, 311 